\title{
Autophagy prevents doxorubicin-induced apoptosis in osteosarcoma
}

\author{
DONGXU ZHAO ${ }^{1}$, HONGPING YUAN ${ }^{2}$, FEI YI ${ }^{1}$, CHUNYANG MENG $^{1}$ and QINGSAN ZHU ${ }^{1}$ \\ ${ }^{1}$ Department of Orthopaedics, China-Japan Union Hospital, Jilin University, Changchun, Jilin 130033; \\ ${ }^{2}$ Department of Nephrology, The Fourth Bethune Hospital, Jilin University, Changchun, Jilin 130021, P.R. China
}

Received November 3, 2013; Accepted February 10, 2014

DOI: $10.3892 / \mathrm{mmr} .2014 .2055$

\begin{abstract}
Autophagy is a process of selective degradation of cellular components. Autophagy is an adaptive process in the majority of tumor cells; it provides sufficient nutrients by degrading cellular components to enhance the survival of tumors. Osteosarcoma is the most common type of primary malignant bone tumor in children and adolescents. Identification of an improved therapeutic strategy for the treatment of osteosarcoma is urgently required. Osteosarcoma has been primarily treated by chemotherapy and the phenomena of resistance to the therapy has become increasingly common. Doxorubicin (Dox) is a classic chemotherapeutic drug for the treatment of osteosarcoma, and certain studies have suggested that Dox induces autophagy. On the basis of the protective effect of autophagy for tumors, the present study investigated whether U2OS and Saos-2 osteosarcoma cells activate autophagy to reduce Dox-induced apoptosis. Dox was observed to inhibit the growth of U2OS and Saos-2 osteosarcoma cells in a concentration-dependent manner. The results of the western blot analysis demonstrated that Dox induced increased expression levels of the apoptosis-related proteins cleaved caspase-3 and cytochrome $c$ and loss of mitochondrial membrane potential (MMP) in the U2OS and Saos-2 osteosarcoma cells. Furthermore, the results of the western blot analysis also revealed that Dox increased the expression levels of the autophagy-related protein microtubule-associated protein 1 light chain 3 and reduced those of p62 in the U2OS and Saos-2 osteosarcoma cells. In order to determine the effect of autophagy on the apoptosis induced by Dox in the U2OS and Saos-2 osteosarcoma cells, autophagy-related protein (Atg)7 small interfering (si) RNA or the autophagy inhibitor 3-methyladenine (3-MA) alone or combined with Dox was used in U2OS and Saos-2 osteosarcoma cells. The results identified that Atg7 siRNA and the autophagy inhibitor
\end{abstract}

Correspondence to: Mr. Qingsan Zhu, Department of Orthopaedics, China-Japan Union Hospital, Jilin University, Changchun, Jilin 130033, P.R. China

E-mail: dr.qszhu@gmail.com

Key words: doxorubicin, osteosarcoma, autophagy, apoptosis
3-MA significantly elevated the levels of growth inhibition by Dox and markedly increased the expression levels of the apoptosis-related proteins cleaved caspase- 3 and cytochrome $c$, and reduced the levels of MMP in the U2OS and Saos-2 osteosarcoma cells, which were treated with Dox. These results indicated that autophagy was the protective mechanism used by U2OS and Saos-2 osteosarcoma against Dox-induced apoptosis. The inhibition of autophagy notably increases the levels of apoptosis induced by Dox. This suggested that Dox used in combination with autophagy inhibitors may effectively treat osteosarcoma.

\section{Introduction}

Osteosarcoma is the most common type of primary malignant bone tumor in children and adolescents. According to a study in 2005, >400 novel cases of pediatric osteosarcoma are diagnosed each year in the USA (1). Osteosarcoma is mainly treated with chemotherapy or surgical excision, but as the prognosis of unresectable or recurrent cases is poor, novel therapies for the treatment of this type of tumor are currently being developed (2). Numerous agents have been used to treat osteosarcoma over the last 30 years and overall survival has exceeded $50 \%$. These agents include high-dose methotrexate, doxorubicin, cisplatin, ifosfamide and etoposide, which has now been sufficient cumulative experience for patients with osteosarcoma (3).

Autophagy is a process of selective degradation of cellular components. There are three major types of autophagy described, which include macroautophagy, microautophagy and chaperone-mediated autophagy (4). At present, the most important type of autophagy is macroautophagy. Macroautophagy, which is referred to as autophagy in the present study, is a nonspecific degradation system that mediates the clearance of long-lived cytoplasmic proteins, including aggregate-prone proteins, certain pathogens and organelles (5-8). In mammalian cells, autophagosome formation begins with a nucleation step, then they expand and fuse to form complete double membrane vesicles termed autophagosomes. Autophagosomes fuse with lysosomes and the contents of the autophagosomes are degraded (9). In this process, two important ubiquitin-like conjugation processes are involved. The first is conjugation of autophagy-related protein (Atg)12 to Atg5 and the second is conjugation of microtubule-associated protein 1 light chain 3 
(LC3; also known as MAP-LC3 or Atg8) to phosphatidylethanolamine (PE), and the two processes are essential for autophagosome formation (10). Notably, autophagy contributes to the maintenance cellular homeostasis and acts as a housekeeping survival mechanism in different harmful conditions, including starvation and endoplasmic reticulum stress (11). A study suggested that autophagy may be important in regulatingof cancer development and progression, and in determining the response of tumor cells to anticancer therapy. However, the role of autophagy in these processes is complicated and depends on the circumstances (12). Autophagy and apoptosis regulate cell fate and are critical in development, normal physiology and in numerous diseases. Although there are more marked differences between these two processes, they can be regulated by the samegene, such as Bcl- (13). A study suggested that there is crosstalk between autophagy and apoptosis in certain situations, for example the autophagy induced by starvation may be inhibited by caspase-mediated cleavage of beclin 1 and the fragment of the cleaved beclin 1 translocates to the mitochondria and induces apoptosis (14). Additionally, autophagic degradation of active caspase-8 inhibits apoptosis (15). Thus, the regulation of apoptosis and autophagy by each other is complicated and the mechanism is unclear.

Dox is a chemotherapeutic agent that activates p53 to induce apoptosis (16). Dox was widely used for treatment of malignancies and exerts a range of effects on the structural and functional properties of tumor cells, ultimately leading to cell death. Although the direct effects of Dox in the damage of DNA have been well studied, the sequence of biochemical events that mediate cell death in response to Dox remains unclear (17).

The present study focused on the role of autophagy in the Dox-induced apoptosis of osteosarcoma cells. The study also investigated whether a combination of autophagy inhibitors and Dox enhanced apoptosis of osteosarcoma cells.

\section{Materials and methods}

Reagents. Atg7 small interfering (si)RNA plasmid was purchased from Santa Cruz Biotechnology, Inc. (Santa Cruz, CA, USA; sc-29918). Dox and 3-methyladenine (3-MA) were purchased from Sigma-Aldrich (St. Louis, MO, USA). Fetal bovine serum (FBS) and Dulbecco's modified Eagle's medium (DMEM) were purchased from Gibco-BRL (Carlsbad, CA, USA). The antibodies anti-nucleoporin p62 antibody (sc-25523; secondary antibody rabbit) anti- cytochrome c antibody (sc-13156; secondary antibody mouse), anti-MAP LC3 $\beta$ antibody (sc-376404; secondary antibody mouse), anti-caspase-3 antibody (sc-65496; secondary antibody mouse), cleaved caspase-3 p11 antibody (sc-22171-R; secondary antibody rabbit) and ATG7 antibody (sc-33211; secondary antibody rabbit) were all purchased from Santa Cruz Biotechnology, Inc.

Cell proliferation assays. U2OS and Saos-2 human cells (ATCC, Rockville, MD, USA) were grown in DMEM with $10 \%$ FBS and cultured at $37^{\circ} \mathrm{C}$ in $5 \% \mathrm{CO}_{2}$. The cell viability was determined by a MTT $[12 \mu 1,5 \mathrm{mg} / \mathrm{ml}$ in phosphate-buffered saline (PBS)] assay. The U2OS and Saos-2 human cells were cultured at a density of $1-1.5 \times 10^{4}$ cells/well in 96 -well plates. Each group was replicated in six separate wells. DMSO was used for the untreated/control cells. Following treatment $(0$,
100,250 or $500 \mathrm{nM}$ ) Dox, the MTT reagent (Sigma-Aldrich) was added to each well for $4 \mathrm{~h}$. Subsequently, the contents of each well was dissolved in $150 \mu 1$ dimethylsulfoxide. The absorbance was recorded at a wavelength of $490 \mathrm{~nm}$ using an ELISA reader (BD Biosciences, Franklin Lake, NJ, USA).

Transfection. Prior to the transfection, the cells were grown to $40 \%$ confluence in each dish. According to the manufacturer's instructions, the U2OS and Saos-2 cells were transfected with $40 \mathrm{nmol} / 1$ control siRNA or Atg7 siRNA using Lipofectamine RNAiMAX reagent (Invitrogen Life Technologies, Carlsbad, CA, USA). After $36 \mathrm{~h}$, the cells were harvested for western blotting.

Western blot analysis. For each assay, the U2OS and Saos-2 cells were washed with cold PBS twice and then $120 \mu 1$ radioimmunoprecipitation assay buffer [ $50 \mathrm{mM}$ Tris- $\mathrm{HCl}, \mathrm{pH} 6.8 ; 0.1 \%$ SDS, $150 \mathrm{mM} \mathrm{NaCl}, 1 \mathrm{mM}$ EDTA, $0.1 \mathrm{mM} \mathrm{Na} \mathrm{VO}_{4}, 1 \mathrm{mM}$ sodium fluoride (NaF), $1 \%$ Triton X-100, $1 \%$ NP-40, $1 \mathrm{mM}$ dithiothreitol, $1 \mathrm{mM}$ PMSF, $1 \mu \mathrm{g} / \mathrm{ml}$ aprotinin, $1 \mu \mathrm{g} / \mathrm{ml}$ leupeptin and $1 \mu \mathrm{g} / \mathrm{ml}$ pepstatin $\mathrm{A}]$ was added to each dish following treatment. The lysates were harvested into $1.5-\mathrm{ml}$ tubes and agitated in a cold room $\left(4^{\circ} \mathrm{C}\right)$ for $20 \mathrm{~min}$. Subsequently, the cell lysates were centrifuged at $13,000 \mathrm{x} \mathrm{g}$ for $15 \mathrm{~min}$, and then the supernatants were harvested. The protein concentrations were detected by a bicinchoninic acid assay (Sigma Aldrich). A total of $60 \mu \mathrm{g}$ protein was used for the western blotting. The lysates were separated by $10 \%(\mathrm{w} / \mathrm{v})$ SDS-polyacrylamide gel electrophoresis. Subsequently, the proteins were transferred onto polyvinylidene difluoride membranes and they were blocked with $5 \%(\mathrm{w} / \mathrm{v})$ skimmed milk in buffer [10 mM Tris- $\mathrm{HCl}(\mathrm{pH} 7.6), 100 \mathrm{mM}$ $\mathrm{NaCl}$ and $0.1 \%(\mathrm{v} / \mathrm{v})$ Tween-20] for $30 \mathrm{~min}$ at room temperature $\left(25^{\circ} \mathrm{C}\right)$. The membranes were incubated in the primary antibodies overnight in a cold room. The following day, the membranes were washed three times with Tris-buffered saline and Tween-20. Subsequently, the membranes were incubated with the secondary antibodies for $1 \mathrm{~h}$ at room temperature. The semi-quantitation of the proteins was analyzed with a Tanon Gel Imager system (Tanon, Shanghai, China).

Mitochondrial membrane potential (MMP) analysis. JC-1 staining to detect the MMP of each group was conducted by flow cytometry, according to the manufacturer's instructions (Molecular Probes, Invitrogen Life Technologies, Carlsbad, CA, USA). Following treatment, the U2OS and Saos-2 cells were trypsinized, washed with PBS, and resuspended in PBS at a concentration of $1 \times 10^{6}$ cells $/ \mathrm{ml}$. The U2OS and Saos-2 cells were then stained with $2.5 \mu 1 \mathrm{JC}-1(1 \mathrm{mg} / \mathrm{ml})$ and incubated in the dark at $37^{\circ} \mathrm{C}$ for $1.5 \mathrm{~h}$. The JC-1 positive cells were subsequently detected by a FACSCalibur flow cytometer (BD Biosciences).

Statistical analysis. Data are representative of three independent experiments and were analyzed by t-test. $\mathrm{P}<0.05$ was considered to indicate a statistically significant difference.

\section{Results}

Dox inhibits the growth of U2OS and Saos-2 osteosarcoma cells in a dose-dependent manner. Dox is widely used for 
A

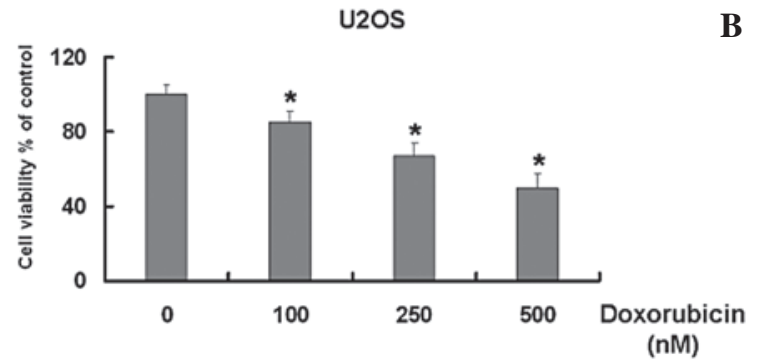

B

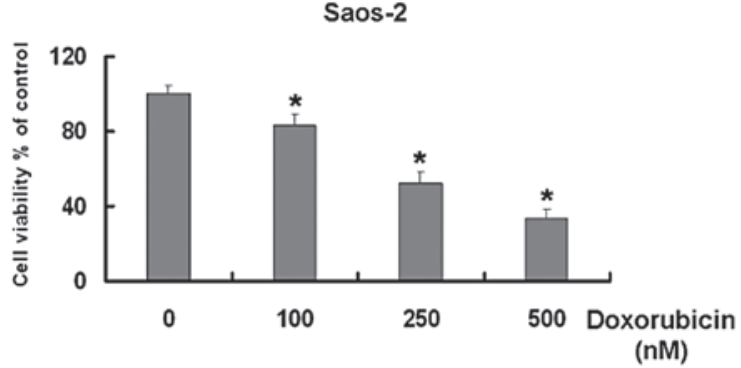

Figure 1. Doxorubicin inhibits the growth of U2OS and Saos-2 cells. (A) U2OS cells and (B) Saos-2 cells were treated with 0, 100,250 and 500 nM doxorubicin for $24 \mathrm{~h}$. Cell viability was detected by an MTT assay. ${ }^{*} \mathrm{P}<0.05$ versus the control group. Data are presented as the mean \pm standard deviation, $\mathrm{n}=6$.

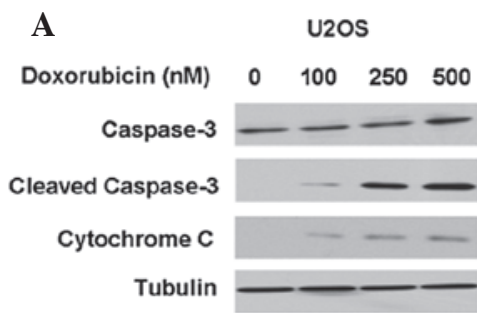

D

Saos-2

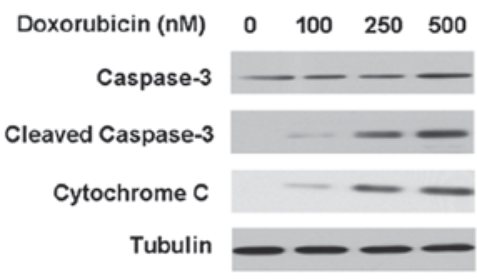

B

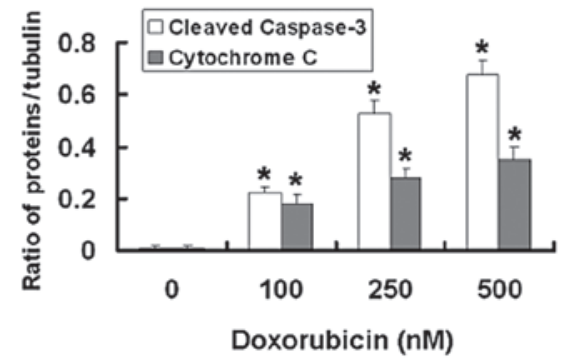

$\mathbf{E}$

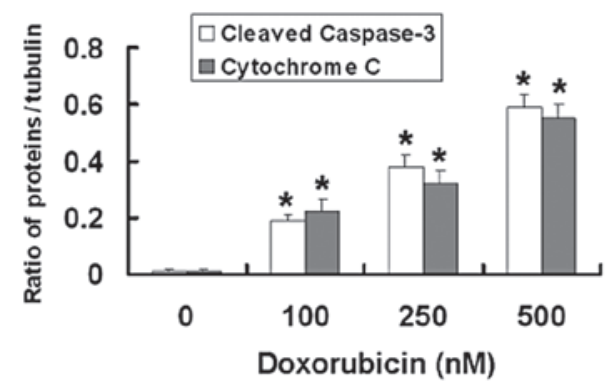

C

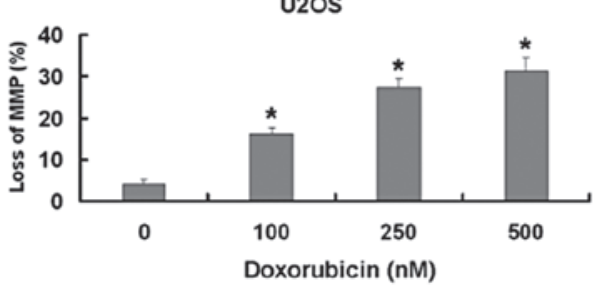

F

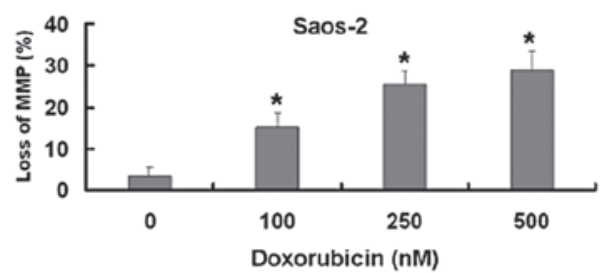

Figure 2. Doxorubicin induces apoptosis in U2OS and Saos-2 cells. (A) Western blot analysis for the expression levels of caspase-3, cleaved caspase-3 and cytochrome $c$ in U2OS cells treated with $0,100,250$ and $500 \mathrm{nM}$ doxorubicin for $24 \mathrm{~h}$. (B) Quantitation of cleaved caspase-3 and cytochrome $c$ protein expression levels in U2OS cells treated with 0, 100, 250 and $500 \mathrm{nM}$ doxorubicin for $24 \mathrm{~h}$. (C) U2OS cells treated with 0, 100, 250 and $500 \mathrm{nM}$ doxorubicin for $24 \mathrm{~h}$. Following treatment, the cells were stained with JC-1. (D) Western blot analysis for the expression levels of caspase-3, cleaved caspase-3 and cytochrome $c$ in Saos-2 cells treated with 0,100,250 and $500 \mathrm{nM}$ doxorubicin for $24 \mathrm{~h}$. (E) Quantitation of cleaved caspase-3 and cytochrome $c$ protein expression levels in Saos-2 cells treated with 0, 100, 250 and $500 \mathrm{nM}$ doxorubicin for $24 \mathrm{~h}$. (F) Saos-2 cells treated with 0, 100, 250 and $500 \mathrm{nM}$ doxorubicin for $24 \mathrm{~h}$. Following treatment, the cells were stained with JC-1. "P<0.05 versus the control group. Data are presented as the mean \pm standard deviation, $\mathrm{n}=3$. MMP, mitochondrial membrane potential.

the treatment of numerous types of tumor (17-21). Initially, the study aimed to identify whether Dox inhibits the growth of the U2OS and Saos-2 osteosarcoma cell lines. The MTT results demonstrated that Dox reduced the cell viability of the two U2OS and Saos-2 cell types in dose-dependent manner compared with that of the untreated cells (Fig. 1).

Dox induces apoptosis in U2OS and Saos-2 cells. As mentioned previously, Dox inhibited the cell growth of the two osteosarcoma cell lines. Subsequently, the levels of apoptosis-associated proteins in the U2OS and Saos-2 cells treated with Dox were detected to investigate whether growth inhibition was associated with apoptosis. The levels of the mitochondrial apoptosis-associated proteins caspase- 3 and cytochrome $c$ were detected.

As shown in Fig. 2A and B, Dox increased the expression levels of cleaved caspase-3 and cytochrome $c$ in the U2OS cells compared with those in the untreated cells. A second osteosarcoma cell line, Saos-2, was used to confirm the aforementioned results. Similar results were observed in the Saos-2 cells (Fig. 2D and E). Furthermore, the MMP was measured in the two cell lines by flow cytometry. As shown in Fig. 2C and F, Dox induced a significant loss in the MMP of the two cells lines.

These results indicated that Dox induces apoptosis in osteosarcoma cells through the mitochondrial apoptotic pathway.

Dox induces autophagy in U2OS and Saos-2 cells. In addition to apoptosis, it has been reported that Dox induces autophagy in tumor cells (22). Thus, the levels of autophagy-associated proteins in the U2OS and Saos-2 cells treated with Dox were subsequently detected. LC3 (the mammalian equivalent of yeast Atg8) and p62 are the two major markers of autophagy. When autophagy occurs, the quantity of LC3-II increases 
A

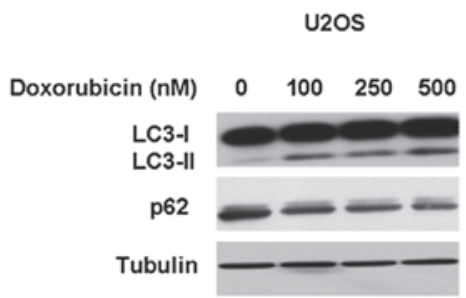

C

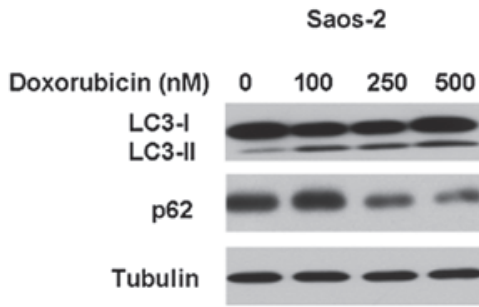

B

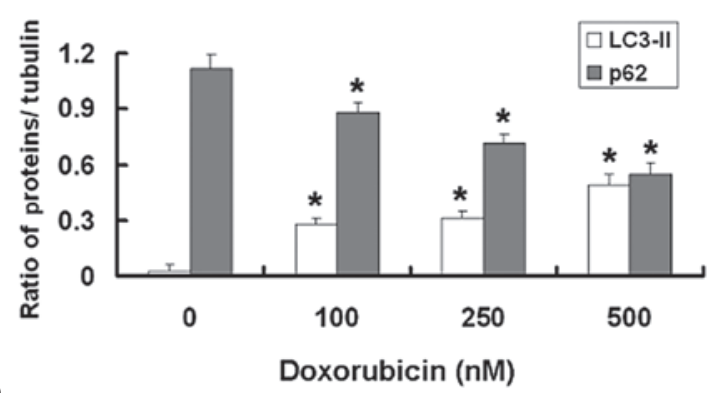

D

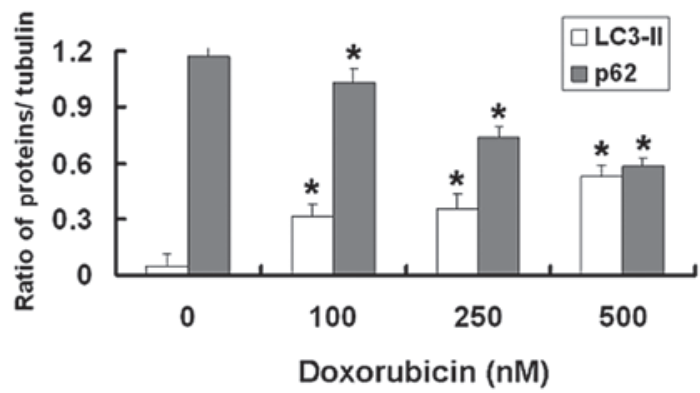

Figure 3. Doxorubicin induces autophagy in U2OS and Saos-2 cells. (A) Western bloting for the expression levels of LC3 and p62 in U2OS cells treated with $0,100,250$ and $500 \mathrm{nM}$ doxorubicin for $24 \mathrm{~h}$. (B) Quantitation of LC3-II and p62 protein expression levels in U2OS cells treated with 0, 100,250 and $500 \mathrm{nM}$ doxorubicin for $24 \mathrm{~h}$. (C) Western blot analysis for the expression levels of LC3 and p62 in Saos-2 cells treated with 0, 100,250 and 500 nM doxorubicin for 24 h. (D) Quantitation of LC3-II and p62 protein expression levels in Saos-2 cells treated with 0, 100, 250 and $500 \mathrm{nM}$ doxorubicin for $24 \mathrm{~h}$. ${ }^{*} \mathrm{P}<0.05$ versus the control group. Data are presented as the mean \pm standard deviation, $n=3$. LC3, microtubule-associated protein 1 light chain 3.
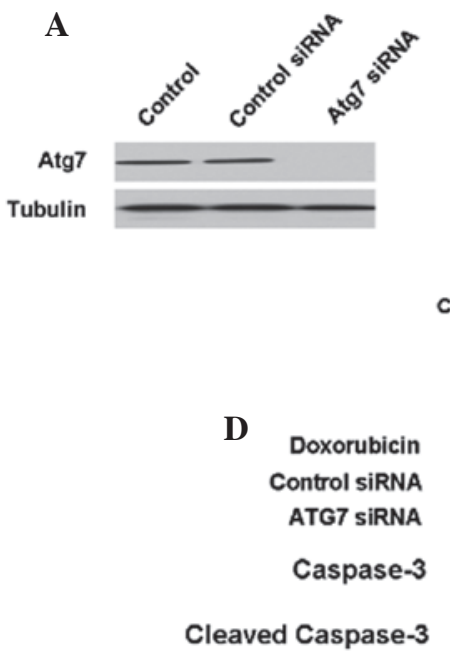

Cytochrome C

Tubulin
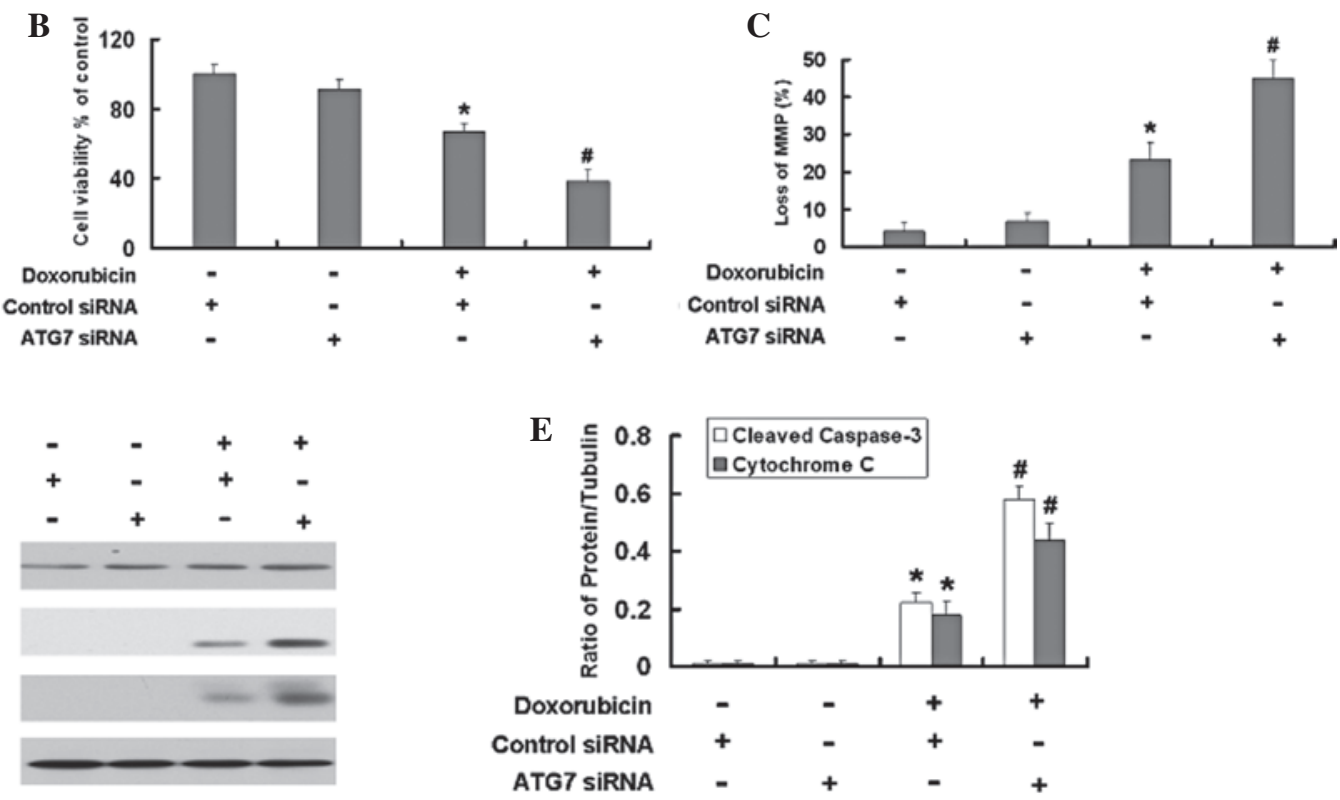

Figure 4. Inhibition of autophagy by Atg7 siRNA enhances apoptosis induced by doxorubicin in U2OS cells. (A) Western blot analysis for the expression levels of Atg7 in U2OS cells treated by control siRNA or Atg7 siRNA for $24 \mathrm{~h}$. (B) U2OS cells treated by control siRNA or Atg7 siRNA with or without doxorubicin for $24 \mathrm{~h}$. Cell viability was detected by an MTT assay. Data are presented as the mean \pm standard deviation, $\mathrm{n}=6$. (C) U2OS cells treated by control siRNA or Atg7 siRNA with or without doxorubicin $(250 \mathrm{nM}, 24 \mathrm{~h})$. Following treatment, the cells were stained with JC-1. (D) Western blot analysis for the expression levels of caspase-3, cleaved caspase-3 and cytochrome $c$ in U2OS cells treated by control siRNA or Atg7 siRNA with or without doxorubicin (250 nM, $24 \mathrm{~h}$ ). (E) Quantitation of cleaved caspase-3 and cytochrome $c$ protein expression levels. ${ }^{*} \mathrm{P}<0.05$ versus the control group; ${ }^{\prime} \mathrm{P}<0.05$ versus the doxorubicin group. Data are presented as the mean \pm standard deviation, $\mathrm{n}=3$. Atg, autophagy-related protein; siRNA, small interfering RNA.

and the expression levels of p62 decrease. Western blotting demonstrated that the levels of these two proteins in U2OS and Saos- 2 cells treated with Dox were altered compared with those in the untreated cells. As shown in Fig. 3, Dox elevated the expression levels of LC3-II and reduced the expression levels of p62 in U2OS and Saos-2 cells.
These results indicated that Dox induces autophagy in U2OS and Saos-2 cells.

Inhibition of autophagy by Atg7 siRNA or an autophagy inhibitor enhances the apoptosis induced by Dox in U2OS and Saos-2 cells. Numerous studies have shown that 
A

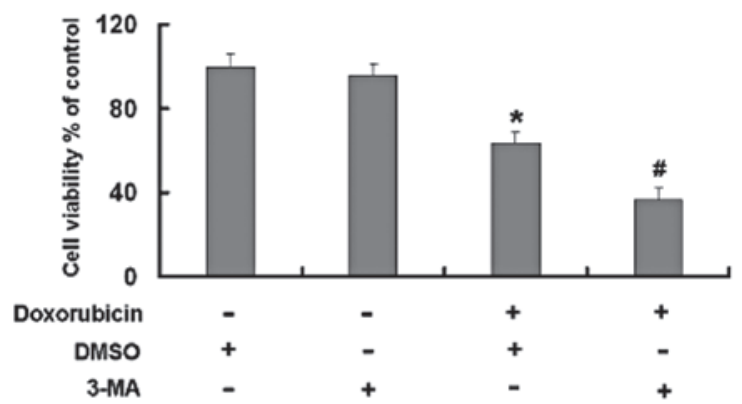

C

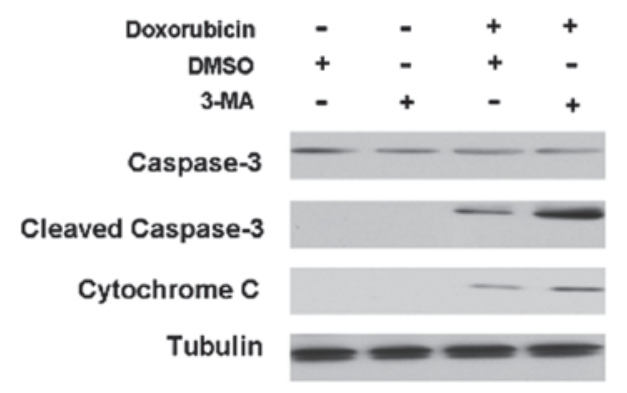

B

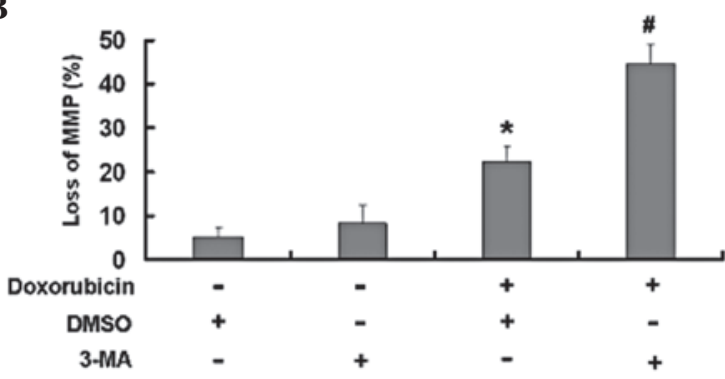

D

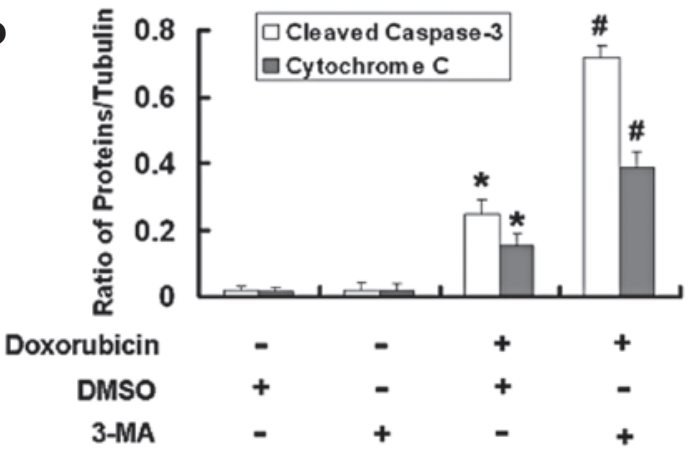

Figure 5. Inhibition of autophagy by the autophagy inhibitor 3-MA enhances apoptosis induced by doxorubicin in U2OS cells. (A) Cell viability was determined by an MTT assay. U2OS cells were treated by doxorubicin (250 nM), 3-MA (10 mM) or doxorubicin and 3-MA for $24 \mathrm{~h}$. Data are presented as the mean \pm standard deviation, $\mathrm{n}=6$. (B) U2OS cells were treated by doxorubicin (250 nM), 3-MA (10 mM) or doxorubicin and 3-MA for 24 h. Following the treatment, the cells were stained with JC-1. (C) Western blot analysis for the expression levels of caspase-3, cleaved caspase-3 and cytochrome $c$ in the cells treated with doxorubicin $(250 \mathrm{nM}), 3-\mathrm{MA}(10 \mathrm{mM})$ or doxorubicin and 3-MA for $24 \mathrm{~h}$. (D) Quantitation of cleaved caspase-3 and cytochrome $c$ protein expression levels. ${ }^{*} \mathrm{P}<0.05$ versus the control group; ${ }^{*} \mathrm{P}<0.05$ versus the doxorubicin group. Data are presented as the mean \pm standard deviation, $\mathrm{n}=3$. $\mathrm{DMSO}$, dimethylsulfoxide; 3-MA, 3-methyladenine; MMP, mitochondrial membrane potential.

autophagy is important in the apoptosis induced by antitumor agents, but the role varies with different agents and types of tumor (12,23). Atg7 siRNA and an autophagy inhibitor were used to investigate the role of autophagy in Dox-induced apoptosis. As shown the Fig. 4A, siAtg7 reduced the expression levels of Atg7 in the U2OS cells compared with those in the control cells. The MTT results demonstrated that Atg7 siRNA intensifies the growth inhibition induced by Dox in the U2OS cells (Fig. 4B). Furthermore, the Atg7 siRNA aggravated the loss of MMP in the U2OS cells (Fig. 4C). Subsequently, the levels of the apoptosis-associated proteins, cleaved capase-3 and cytochrome $c$, were detected. The Atg7 siRNA further increased the expression levels of the apoptosis-associated proteins, cleaved capase- 3 and cytochrome $c$, induced by Dox in the U2OS cells (Fig. 4D and E).

Subsequently, the autophagy inhibitor 3-MA was used to confirm the role of autophagy in Dox-induced apoptosis. The combination of 3-MA and Dox also intensified the growth inhibition of the U2OS cells induced by Dox (Fig. 5A). As shown in Fig. 5B, C and D, the combination of 3-MA and Dox further increased the expression levels of the apoptosis-associated proteins cleaved capase- 3 and cytochrome $c$ and loss of MMP induced by Dox in the U2OS cells.

Saos- 2 cells were used to confirm these results and similar results were observed (Figs. 6 and 7).

These results in the U2OS and Saos-2 cells indicated that autophagy is important in Dox-induced apoptosis and the inhibition of autophagy further increases the cytotoxicity of Dox in osteosarcoma cells.

\section{Discussion}

Osteosarcoma is common type of primary bone tumor. Due to the high metastatic potential and the common acquisition of chemotherapeutic resistance in osteosarcoma, the clinical outcome is poor (18). Chemotherapy for osteosarcoma is administered in neoadjuvant and adjuvant settings. Clinical treatment commonly uses chemotherapy for the treatment of osteosarcoma, in order to avoid amputation. Dox is a common conventional chemotherapeutic drug used for the treatment of osteosarcoma (19). Studies have indicated that Dox inhibits the cell growth of human leukemia cells (17) and breast cancer cell lines (20). In order to investigate the effect of Dox on osteosarcoma, the present study investigated the effect of Dox on the cell viability of two human osteosarcoma cell lines, U2OS and Saos-2, by MTT assay. The results demonstrated that Dox inhibited the cell proliferation of the U2OS and Saos-2 cells in a dose-dependent manner.

A previous study reported that Dox induced apoptosis by increasing the expression levels of cleaved caspase-3 (18). Cleaved caspase- 3 is an activated form of caspase- 3 , and it leads to cell death. Therefore, in order to detect whether Dox induces the apoptosis of the two osteosarcoma cell lines, the expression levels of cleaved caspase- 3 and cytochrome $c$ were detected by western blot analysis of the U2OS and Saos-2 cells. The results showed that Dox increased the expression levels of cleaved caspase- 3 and cytochrome $c$ in a dose-dependent manner in the U2OS and Saos-2 cells. Additionally, the MMP loss was measured in the two cell 

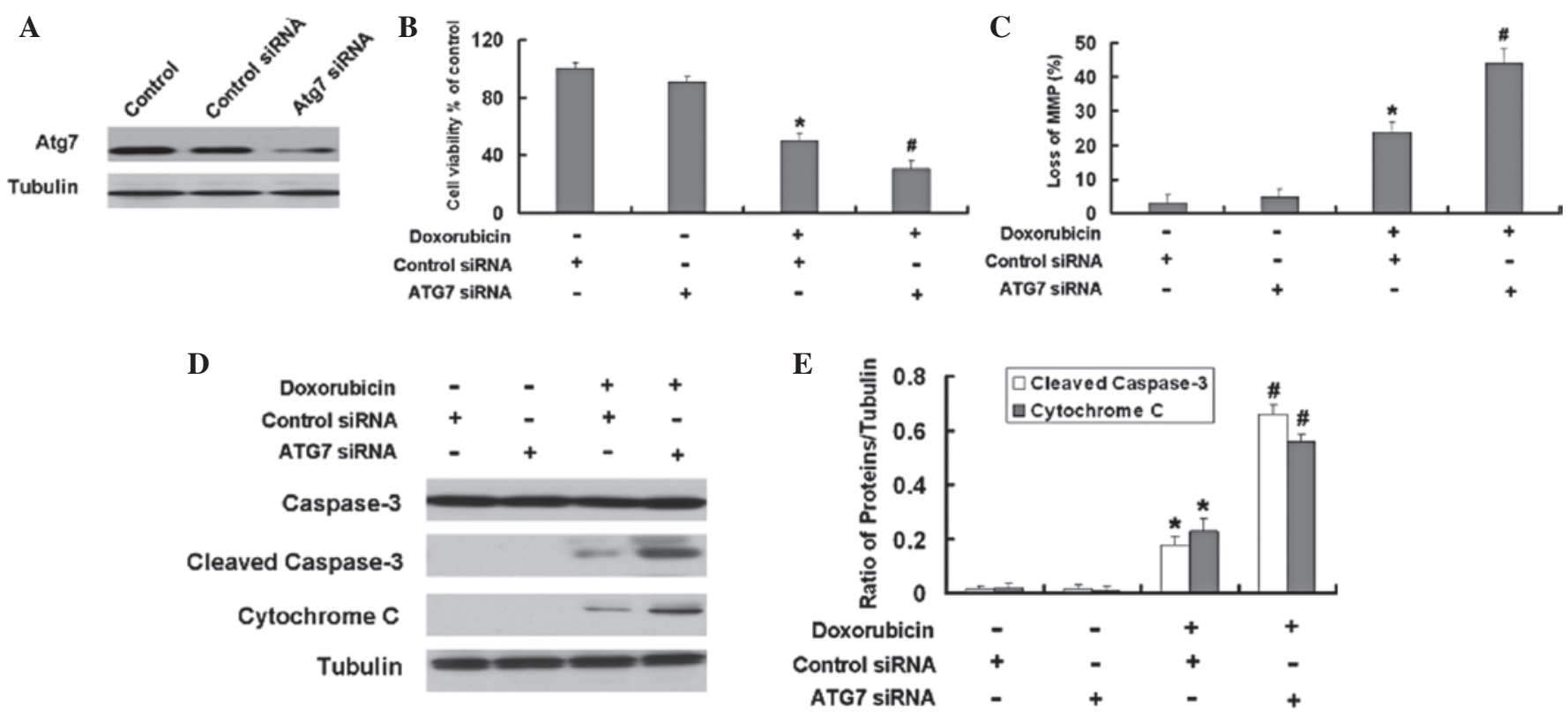

Figure 6. Inhibition of autophagy by Atg7 siRNA enhances apoptosis induced by doxorubicin in Saos-2 cells. (A) Western blot analysis for the expression levels of Atg7 in Saos-2 cells treated with control siRNA or Atg7 siRNA for $24 \mathrm{~h}$. (B) Saos-2 cells treated by control siRNA or Atg7 siRNA with or without doxorubicin for $24 \mathrm{~h}$. Cell viability was detected by an MTT assay. Data are presented as the mean \pm standard deviation, $\mathrm{n}=6$. (C) Saos-2 cells treated by control siRNA or Atg7 siRNA with or without doxorubicin $(250 \mathrm{nM}, 24 \mathrm{~h})$. Following treatment, the cells were stained with JC-1. (D) Western blot analysis for the expression levels of caspase-3, cleaved caspase-3 and cytochrome $c$ in Saos-2 cells treated by control siRNA or Atg7 siRNA with or without doxorubicin (250 nM, 24 h). (E) Quantitation of cleaved caspase- 3 and cytochrome $c$ protein expression levels. ${ }^{*} \mathrm{P}<0.05$ versus the control group; ${ }^{~} \mathrm{P}<0.05$ versus the doxorubicin group. Data are presented as the mean \pm standard deviation, $n=3$. Atg, autophagy-related protein; MMP, mitochondrial membrane potential; siRNA, small interfering RNA.
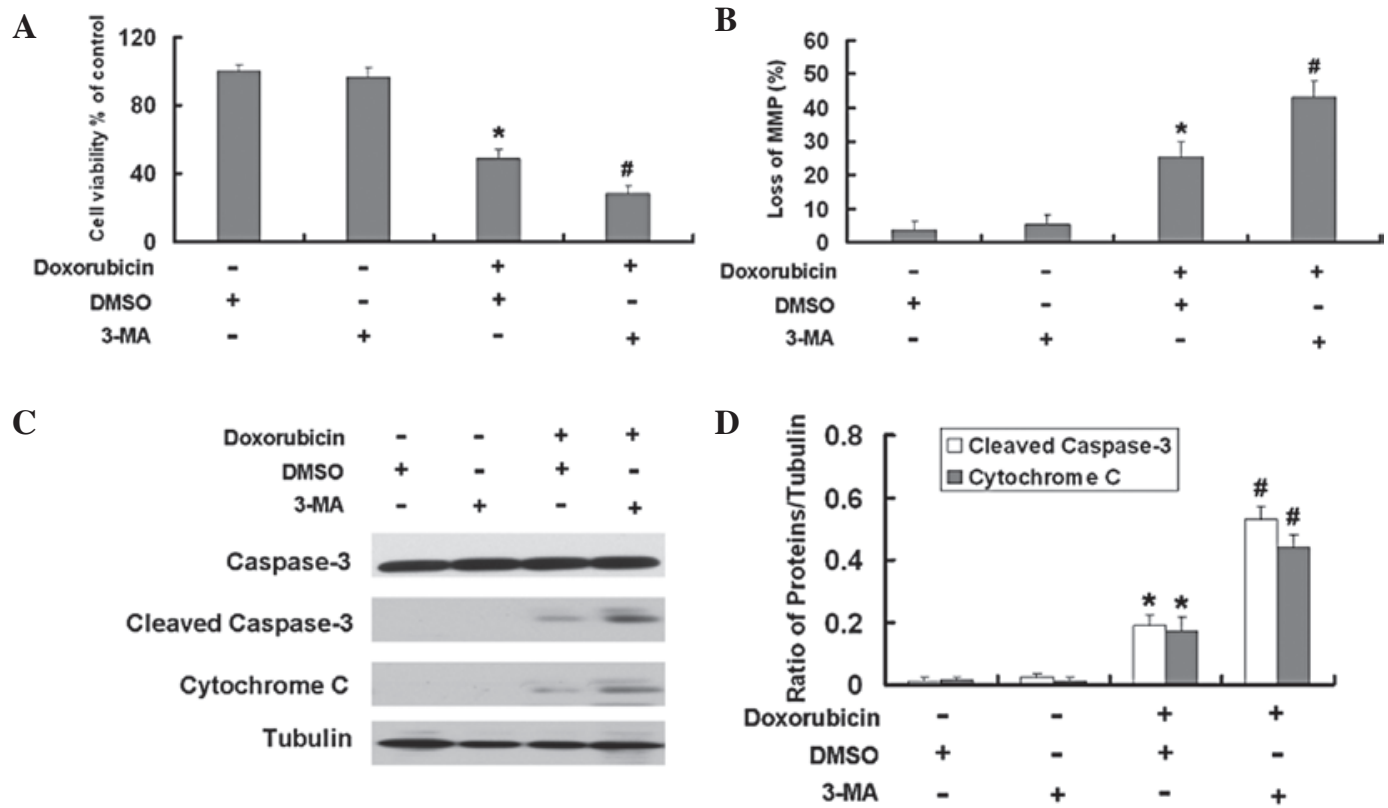

Figure 7. Inhibition of autophagy by the autophagy inhibitor 3-MA enhances apoptosis induced by doxorubicin in Saos-2 cells. (A) Cell viability was determined by an MTT assay. Saos-2 cells were treated with doxorubicin $(250 \mathrm{nM}), 3-\mathrm{MA}(10 \mathrm{mM})$ or doxorubicin and 3-MA for $24 \mathrm{~h}$. Data are presented as the mean \pm standard deviation, $n=6$. (B) Saos-2 cells were treated with doxorubicin $(250 \mathrm{nM}), 3-\mathrm{MA}(10 \mathrm{mM})$ or doxorubicin and 3-MA for $24 \mathrm{~h}$. Following the treatment, the cells were stained with JC-1. (C) Western blot analysis for the expression levels of caspase-3, cleaved caspase-3 and cytochrome $c$ treated with doxorubicin $(250 \mathrm{nM}), 3-\mathrm{MA}(10 \mathrm{mM})$ or doxorubicin and 3-MA for $24 \mathrm{~h}$. (D) Quantitation of cleaved caspase-3 and cytochrome $c$ protein expression levels ${ }^{*} \mathrm{P}<0.05$ versus the control group; ${ }^{\prime} \mathrm{P}<0.05$ versus the doxorubicin group. Data are presented as the mean \pm standard deviation, $\mathrm{n}=3$. DMSO, dimethylsulfoxide; 3-MA, 3-methyladenine; MMP, mitochondrial membrane potential.

lines using flow cytometry. Dox evidently induced the loss of the MMP in the two cells lines.

Although the present study demonstrated that Dox induces apoptosis of U2OS and Saos-2 cells, the effect of the treatment requires enhancement. Therefore, the other functions of Dox were investigated. Fong et al (22) identified that Dox induced increased expression levels of LC3 in the A2780 epithelial ovarian cancer cell line. LC3, a homologue of Apg8p that 
is essential for autophagy in yeast, is a classical marker of autophagy. In particular, LC3-II is the first mammalian protein identified that specifically associates with autophagsome membranes (24). In order to determine whether Dox has an effect on autophagy in U2OS and Saos-2 cells, the expression levels of LC3 were detected by western blotting and it was demonstrated that Dox induced the increased expression levels of LC3 in the U2OS and Saos-2 cells. In addition, Dox reduced the levels of another autophagy marker, p62. All the results showed that Dox induced autophagy in the U2OS and Saos- 2 cells.

A growing number of studies have reported crosstalk between apoptosis and autophagy (25). The correlation between them is complicated. A number of studies have provided evidence that autophagy serves as a survival pathway in tumor cells treated with anticancer drugs and proposed a rationale for the use of autophagy inhibitors in combination with therapies designed to induce apoptosis in human cancers $(26,27)$. Therefore, autophagy inhibition represents a major therapeutic target for chemosensitization (28). Although the present study demonstrated that Dox induced apoptosis and autophagy in U2OS and Saos-2 cells, the correlation between apoptosis and autophagy induced by Dox is unknown. In order to elucidate the correlation, Dox was used in combination with the autophagy inhibitor 3-MA and Atg7 siRNA to detect the cell proliferation and the expression levels of apoptosis-associated proteins induced by Dox in the U2OS and Saos- 2 cells. As expected, the results showed that the autophagy inhibitor 3-MA and Atg7 siRNA significantly enhanced the cell proliferation inhibition and notably increased the expression levels of the apoptosis proteins cleaved caspase-3 and cytochrome $c$ induced by Dox in the U2OS and Saos- 2 cells. These results demonstrated that autophagy is preventative against the apoptosis induced by Dox in U2OS and Saos-2 cells.

The present study provides significant data indicating that inhibition of autophagy may enhance the tumor cell proliferation inhibition and apoptosis of U2OS and Saos-2 cells induced by Dox. Thus, activation of autophagy may be involved in the resistance to apoptosis of U2OS and Saos-2 cells. Autophagy may enable tumor cells adapt to metabolic stress and promote cell survival during apoptosis. In conclusion, the present study showed that inhibition of autophagy may be a novel strategy to increase the efficacy of anticancer drugs in the treatment of U2OS and Saos-2 cells.

\section{References}

1. Chou AJ, Merola PR, Wexler LH, et al: Treatment of osteosarcoma at first recurrence after contemporary therapy: the Memorial Sloan-Kettering Cancer Center experience. Cancer 104: 2214-2221, 2005.

2. Nakase M, Inui M, Okumura K, Kamei T, Nakamura S and Tagawa T: p53 gene therapy of human osteosarcoma using a transferrin-modified cationic liposome. Mol Cancer Ther 4 625-631, 2005

3. Janeway KA and Grier HE: Sequelae of osteosarcoma medical therapy: a review of rare acute toxicities and late effects. Lancet Oncol 11: 670-678, 2010.

4. Yang Z and Klionsky DJ: Eaten alive: a history of macroautophagy. Nat Cell Biol 12: 814-822, 2010.
5. Klionsky DJ: The molecular machinery of autophagy: unanswered questions. J Cell Sci 118: 7-18, 2005.

6. Levine B and Klionsky DJ: Development by self-digestion: molecular mechanisms and biological functions of autophagy. Dev Cell 6: 463-477, 2004.

7. Mizushima N: The pleiotropic role of autophagy: from protein metabolism to bactericide. Cell Death Differ 12 (Suppl 2): $1535-1541,2005$

8. Rubinsztein DC, Gestwicki JE, Murphy LO and Klionsky DJ Potential therapeutic applications of autophagy. Nat Rev Drug Discov 6: 304-312, 2007.

9. Huang WP and Klionsky DJ: Autophagy in yeast: a review of the molecular machinery. Cell Struct Funct 27: 409-420, 2002.

10. Luo S and Rubinsztein DC: Apoptosis blocks Beclin 1-dependent autophagosome synthesis: an effect rescued by Bcl-xL. Cell Death Differ 17: 268-277, 2010.

11. Del Bello B, Toscano M, Moretti D and Maellaro E: Cisplatin-induced apoptosis inhibits autophagy, which acts as a pro-survival mechanism in human melanoma cells. PLoS One 8: e57236, 2013.

12. Hippert MM, O'Toole PS and Thorburn A: Autophagy in cancer: good, bad, or both? Cancer Res 66: 9349-9351, 2006.

13. Thorburn A: Apoptosis and autophagy: regulatory connections between two supposedly different processes. Apoptosis 13: 1-9, 2008.

14. Djavaheri-Mergny M, Maiuri MC and Kroemer G: Cross talk between apoptosis and autophagy by caspase-mediated cleavage of Beclin 1. Oncogene 29: 1717-1719, 2010.

15. Hou W, Han J, Lu C, Goldstein LA and Rabinowich H: Autophagic degradation of active caspase-8: a crosstalk mechanism between autophagy and apoptosis. Autophagy 6: 891-900, 2010.

16. Lowe SW, Ruley HE, Jacks T and Housman DE: p53-dependent apoptosis modulates the cytotoxicity of anticancer agents. Cell 74: 957-967, 1993

17. Yu R, Shtil AA, Tan TH, Roninson IB and Kong AN: Adriamycin activates c-jun $\mathrm{N}$-terminal kinase in human leukemia cells: a relevance to apoptosis. Cancer Lett 107: 73-81, 1996.

18. Spina A, Sorvillo L, Di Maiolo F, et al: Inorganic phosphate enhances sensitivity of human osteosarcoma U2OS cells to doxorubicin via a p53-dependent pathway. J Cell Physiol 228 198-206, 2013

19. Gill J, Ahluwalia MK, Geller D and Gorlick R: New targets and approaches in osteosarcoma. Pharmacol Ther 137: 89-99, 2013

20. Radetzki S, Köhne $\mathrm{CH}$, von Haefen $\mathrm{C}$, et al: The apoptosis promoting Bcl-2 homologues Bak and Nbk/Bik overcome drug resistance in Mdr-1-negative and Mdr-1-overexpressing breast cancer cell lines. Oncogene 21: 227-238, 2002.

21. Tarquini F Tiribuzi R, Crispoltoni L, et al: Caspase 3 activation and PARP cleavage in lymphocytes from newborn babies of diabetic mothers with unbalanced glycaemic control. Cell Biochem Funct 32: 87-95, 2014

22. Fong MY, Jin S, Rane M, Singh RK, Gupta R and Kakar SS Withaferin A synergizes the therapeutic effect of doxorubicin through ROS-mediated autophagy in ovarian cancer. PLoS One 7: e42265, 2012

23. Li H, Jin X, Zhang Z, Xing Y and Kong X: Inhibition of autophagy enhances apoptosis induced by the PI3K/AKT/ mTor inhibitor NVP-BEZ235 in renal cell carcinoma cells. Cell Biochem Funct 31: 427-433, 2013.

24. Kabeya Y, Mizushima N, Ueno T, et al: LC3, a mammalian homologue of yeast Apg8p, is localized in autophagosome membranes after processing. EMBO J 19: 5720-5728, 2000.

25. Kang R, Zeh HJ, Lotze MT and Tang D: The Beclin 1 network regulates autophagy and apoptosis. Cell Death Differ 18: 571-580, 2011.

26. Zhong JT, Xu Y, Yi HW et al: The BH3 mimetic S1 induces autophagy through ER stress and disruption of Bcl-2/Beclin 1 interaction in human glioma U251 cells. Cancer Lett 323: 180-187, 2012.

27. Chang Z, Shi G, Jin J, et al: Dual PI3K/mTOR inhibitor NVP-BEZ235-induced apoptosis of hepatocellular carcinoma cell lines is enhanced by inhibitors of autophagy. Int J Mol Med 31: 1449-1456, 2013.

28. Fimia GM and Piacentini M: Regulation of autophagy in mammals and its interplay with apoptosis. Cell Mol Life Sci 67: $1581-1588,2010$ 